\title{
Culture-Positive Spontaneous Ascitic Infection in Patients with Acute Decompensated Cirrhosis: Multidrug-Resistant Pathogens and Antibiotic Strategies
}

\author{
Jing Liu ${ }^{1,2}$, Yanhang $\mathrm{Gao}^{2,3}$, Xianbo Wang ${ }^{2,4}$, Zhiping Qian ${ }^{2,5}$, Jinjun Chen ${ }^{2,6}$, Yan Huang ${ }^{2,7}$, Zhongji Meng ${ }^{2,8}$, \\ Xiaobo Lu ${ }^{2,9}$, Guohong Deng ${ }^{2,10}$, Feng Liu' ${ }^{2,11}$, Zhiguo Zhang ${ }^{12}$, Hai Li ${ }^{2,13}$, and Xin Zheng ${ }^{1,2}$ \\ ${ }^{1}$ Department of Infectious Diseases, Institute of Infection and Immunology, Union Hospital, Tongji Medical College, \\ Huazhong University of Science and Technology, Wuhan; \\ ${ }^{2}$ Chinese Acute-on-Chronic Liver Failure Consortium, CATCH-LIFE, Shanghai; \\ ${ }^{3}$ Department of Hepatology, The First Hospital of Jilin University, Changchun; \\ ${ }^{4}$ Center of Integrative Medicine, Beijing Ditan Hospital, Capital Medical University, Beijing; \\ ${ }^{5}$ Department of Liver Intensive Care Unit, Shanghai Public Health Clinical Centre, Fudan University, Shanghai; \\ ${ }^{6}$ Hepatology Unit, Department of Infectious Diseases, Nanfang Hospital, Southern Medical University, Guangzhou; \\ ${ }^{7}$ Department of Infectious Disease, Hunan Key Laboratory of Viral Hepatitis, Xiangya Hospital, Central South University, Changsha; \\ ${ }^{8}$ Department of Infectious Disease, Taihe Hospital, Hubei University of Medicine, Shiyan; \\ ${ }^{9}$ Liver Disease Center, First Affiliated Hospital of Xinjiang Medical University, Urumuqi; \\ ${ }^{10}$ Department of Infectious Diseases, Southwest Hospital, Third Military Medical University (Army Medical University), Chongqing; \\ ${ }^{11}$ Department of Infectious Diseases and Hepatology, The Second Hospital of Shandong University, Jinan; \\ ${ }^{12}$ School of Medicine and Health Management, Tongji Medical College of Huazhong University of Science and Technology, Wuhan; \\ ${ }^{13}$ Department of Gastroenterology, Ren Ji Hospital, School of Medicine, Shanghai Jiao Tong University, Shanghai, China.
}

\begin{abstract}
Purpose: This study investigated multidrug-resistant (MDR) pathogens and antibiotic strategies of culture-positive spontaneous ascitic infection (SAI) in patients with acute decompensated cirrhosis.

Materials and Methods: We retrospectively analyzed 432 acute decompensated cirrhotic patients with culture-positive SAI from 11 teaching hospitals in China (January 2012 to May 2018). A Cox proportional hazards model analysis was conducted to identify independent predictors of 28-day mortality.

Results: A total of 455 strains were isolated from 432 ascitic culture samples. Gram-negative bacteria (GNB), gram-positive bacteria (GPB), and fungi caused 52.3, 45.5, and 2.2\% of all SAI episodes, respectively. Episodes were classified as nosocomial (41.2\%), healthcare-related (34.7\%), and community-acquired (24.1\%). Escherichia coli (13.4\%) and Klebsiella pneumoniae (2.4\%) were extendedspectrum $\beta$-lactamase producing isolates. The prevalence of methicillin-resistant Staphylococcus aureus was 1.1\%. Ceftazidime, cefepime, aztreonam, and amikacin were recommended as first-line antibiotics agents for non-MDR GNB infections; piperacillin/tazobactam and carbapenems for MDR GNB in community-acquired and healthcare-related or nosocomial infections, respectively; and vancomycin or linezolid for GPB infections, regardless of drug-resistance status. Multivariate analysis revealed days of hospital stay before SAI, upper gastrointestinal bleeding, white blood cell count, alanine aminotransferase, serum creatinine concentration, total bilirubin, and international normalized ratio as key independent predictors of 28-day mortality.

Conclusion: MDR pathogens and antibiotic strategies were identified in patients with acute decompensated cirrhosis with culture-positive SAI, which may help optimize therapy and improve clinical outcomes.
\end{abstract}

Key Words: Spontaneous ascitic infection, cirrhosis, multidrug-resistant, antibiotic strategies, risk factors

Received: September 27, 2019 Revised: December 13, 2019 Accepted: December 16, 2019

Corresponding author: Xin Zheng, MD, PhD, Department of Infectious Diseases, Institute of Infection and Immunology, Union Hospital, Tongji Medical College, Huazhong University of Science and Technology, Wuhan, Hubei 430022, China.

Tel: 86-027-85726978, Fax: 86-027-85726398, E-mail: xin11@hotmail.com

-The authors have no potential conflicts of interest to disclose

(C) Copyright: Yonsei University College of Medicine 2020

This is an Open Access article distributed under the terms of the Creative Commons Attribution Non-Commercial License (https://creativecommons.org/licenses/by-nc/4.0) which permits unrestricted non-commercial use, distribution, and reproduction in any medium, provided the original work is properly cited. 


\section{INTRODUCTION}

Liver cirrhosis is a global health and economic burden, causing significant mortality. ${ }^{1}$ One of the severe complications in patients with cirrhosis is bacterial infection. ${ }^{2}$ Bacterial infection is a major cause of acute decompensation in patients with cirrhosis and has been shown to be a trigger for developing acuteon-chronic liver failure in acute decompensated cirrhosis patients, which is a key prognostic determinant and is significantly associated with mortality. ${ }^{3,4}$

Spontaneous ascitic infection (SAI) is defined as an ascitic fluid infection without an obvious intra-abdominal contiguous source of infection and is one of the most common infections in patients with cirrhosis. ${ }^{5}$ Patients with SAI can be classified into two groups: 1) Culture-negative neutrocytic ascites is diagnosed based on an ascitic fluid polymorphonuclear leukocyte (PMN) count $\geq 250 / \mathrm{mm}^{3}$ with a negative culture. ${ }^{6}$ For these patients, antibiotic treatment is based on the physician's experience. 2) Culture-positive SAI includes spontaneous bacterial peritonitis (SBP) and bacterascites. The prognosis and severity of liver insufficiency in patients with SBP and bacterascites are highly comparable, and the 30-day mortality rates of these two types of patients are reported as $31.5 \%$ and $32 \%$, respectively. ${ }^{7.8}$

Early SAI diagnosis contributes to effective antibiotic therapy and improved survival. ${ }^{9}$ However, successful empirical antibiotic therapy must be based on local epidemiologic data. Previous studies have reported that gram-negative bacteria (GNB), mainly Enterobacteriaceae, are the major causative organisms of ascites. ${ }^{10}$ While third-generation cephalosporins have been used as the first-line treatment for SBP for several years, ${ }^{11}$ recently, an increase in multidrug-resistant (MDR) agents, such as extended spectrum $\beta$-lactamase (ESBL)-producing Enterobacteriaceae, have been observed in healthcare-associated and nosocomial infections, leading to failure of antibiotic strategies based on third-generation cephalosporins or quinolones and poor outcomes. ${ }^{12}$ Therefore, the European Association for the Study of the Liver (EASL) lately updated their recommendations of empirical antibiotic therapy for patients with $\mathrm{SBP} .{ }^{13}$ However, because of various antibiotic prescription patterns in different regions and microbiological changes over time, the prevalence of antibiotic-resistant pathogens and the pathogen spectrum substantially differ geographically, ${ }^{3,14}$ and more evidence is required to support the application of empirical antibiotic management based on the EASL guidelines for cirrhotic patients with SBP to other regions. Thus, further studies with a larger sample size on bacteriological and drug resistance of culture-positive SAI are extremely urgent.

Cirrhotic patients with SBP are nearly 1.5-fold more likely to die within 28 days than those with non-SBP infection. ${ }^{15}$ Two epidemiological studies of SBP have shown that delay in diagnostic paracentesis and inadequate treatment were independent predictors of mortality associated with SBP. ${ }^{16,17}$ However, few studies have evaluated the specific risk factors for SAI mortality in patients with culture-positive acute decompensated cirrhosis. Therefore, this study aimed to assess possible changes in the pathogen spectrum and in drug resistance in patients with acute decompensated cirrhosis with culture-positive SAI based on site of infection acquisition and to identify independent risk factors of 28-day mortality after the first culture-positive SAI episode.

\section{MATERIALS AND METHODS}

\section{Study design}

We retrospectively studied patients admitted to 11 different teaching hospitals diagnosed with acute decompensated cirrhosis with culture-positive SAI from January 1, 2012 to May 30,2018 . The clinical history, physical examination, and laboratory and microbiological results were recorded in a pre-established electronic chart. For patients who presented with multiple culture-positive SAI during the study period, only the first episode was considered for analysis. The study was approved by the Ethics Committee of Tongji Medical College, Huazhong University of Science and Technology, and the ethical committees of each center (IORG No: IORG0003571).

\section{Definitions}

Diagnosis of liver cirrhosis was established by ultrasonographic findings, CT, MRI, and biopsy. ${ }^{12}$ Acute decompensation was defined as acute development of one or more major complications of liver disease, including acute development of large ascites (within less than 2 weeks; ultrasound ascites volume $\geq 150$ $\mathrm{mL}$ ), acute hepatic encephalopathy, acute gastrointestinal hemorrhage, and bacterial infection. ${ }^{4}$ Definition of organ failure was based on the chronic liver failure consortium organ failure score. ${ }^{4}$ SAI was classified as nosocomial if the infection was diagnosed beyond $48 \mathrm{~h}$ of admission; as healthcare-associated if diagnosis was made within $48 \mathrm{~h}$ of admission in patients requiring hospitalization for at least 2 days or paying more than two visits to the emergency department in the previous 90 days prior to infection or receiving chemotherapy/hemodialysis in the previous 30 days preceding the infection; and as community-acquired in all other cases. ${ }^{18} \mathrm{SBP}$ was defined as an ascitic fluid PMN count $\geq 250 / \mathrm{mm}^{3}$ with a positive culture result. Bacterascites was defined as a positive bacterial culture with ascitic fluid PMN count $<250 / \mathrm{mm}^{3}$. Definitions of blood stream infections, urinary tract infections, and skin and soft tissue infections were based on other studies. ${ }^{19}$ Resistant microorganisms were defined as MDR if they demonstrated non-susceptibility to at least one agent in three and as extensively drug resistant (XDR) in case of nonsusceptibility to at least one agent in all but two or fewer antimicrobial categories. ${ }^{20}$

\section{Microbiology}

Identification and susceptibility tests of the isolated pathogens 
Table 1. Clinical Characteristics of Patients with Community-Acquired, Healthcare-Associated, and Nosocomial Culture-Positive Spontaneous Ascitic Infections

\begin{tabular}{|c|c|c|c|c|}
\hline & Community-acquired ( $n=104$ ) & Healthcare-associated ( $n=150)$ & Nosocomial (n=178) & $p$ value \\
\hline Age (yr) & $55 \pm 11.5$ & $56 \pm 12.6$ & $56 \pm 11.6$ & 0.419 \\
\hline Male/female & $80 / 24$ & $107 / 43$ & $125 / 53$ & 0.460 \\
\hline Exposure to health care services* & $22(21.2)$ & 89 (59.3) & $93(52.2)$ & $<0.001$ \\
\hline Previous antibiotics uses ${ }^{\dagger}$ & $2(1.9)$ & $33(22.0)$ & 30 (16.9) & $<0.001$ \\
\hline Serum WBC ( $\left.\times 10^{9} / L\right)$ & $5.2(3.2-8.5)$ & $6.8(4.3-10.7)$ & $6.8(4.1-10.0)$ & 0.011 \\
\hline Neutrophil $\left(\times 10^{9} / \mathrm{L}\right)$ & $3.7(1.9-6.6)$ & $5.2(2.6-8.9)$ & $4.7(2.8-8.0)$ & 0.014 \\
\hline PLT $\left(\times 10^{9} / \mathrm{L}\right)$ & $88(56.3-114.7)$ & 76 (49.9-113.8) & $73(48.8-102.0)$ & 0.233 \\
\hline AST (U/L) & $53.3(32.6-87.4)$ & $54.2(35.3-98.4)$ & $44.0(29.5-83.9)$ & 0.155 \\
\hline ALT ( U/L) & $29.4(19.0-65.6)$ & $35.5(22.0-66.2)$ & $27.0(17.0-54.4)$ & 0.109 \\
\hline Total bilirubin (mg/dL) & $3.0(1.5-7.1)$ & $4.1(2.1-9.1)$ & $3.8(1.8-9.1)$ & 0.077 \\
\hline Serum creatinine (mg/dL) & $0.9(0.7-1.3)$ & $1.0(0.7-1.6)$ & $1.0(0.7-1.4)$ & 0.535 \\
\hline Albumin (g/L) & $26.5 \pm 8.0$ & $28.0 \pm 10.5$ & $28.8 \pm 5.4$ & 0.071 \\
\hline INR & $1.6 \pm 0.5$ & $1.9 \pm 0.8$ & $1.8 \pm 0.8$ & 0.016 \\
\hline MELD & $15 \pm 8.6$ & $18 \pm 9.8$ & $17 \pm 9.4$ & 0.061 \\
\hline MELD-Na & $21 \pm 7.1$ & $24 \pm 7.9$ & $23 \pm 7.7$ & 0.001 \\
\hline Child-Pugh score & $10 \pm 2.0$ & $11 \pm 2.0$ & $10 \pm 1.9$ & 0.175 \\
\hline 28-day mortality & $14(13.5)$ & 41 (27.3) & 34 (19.1) & 0.022 \\
\hline
\end{tabular}

WBC, white blood cell; PLT, platelet; AST, aspartate transaminase; ALT, alanine aminotransferase; INR, international normalized ratio; MELD, Model for EndStage Liver Disease; MELD-Na, Model for End-Stage Liver Disease Sodium.

Categorical variables: number (percentage). Continuous variables: means $\pm S D$, median and interquartile ranges.

*Health care services are defined as hospitalization or contact with the health care system (emergency department, nursing home, and clinic) within 2 weeks prior to admission. 'Previous antibiotics are defined as any antibiotics used for more than 2 days during the 14 days before admission.

fections were significantly and positively correlated with nonsurvivors ( $p=0.007)$. Similarly, MDR organisms were isolated more frequently from non-survivor groups with $\mathrm{GNB}(p=0.267)$ and GPB ( $p=0.455)$ infections (Table 2).

We further investigated the distributions of GNB and GPB between SBP and bacterascites groups. The results showed that GNB were the major pathogens in the SBP group (71.6\%), whereas GPB were predominant in the bacterascites group (57.3\%), and the differences in GNB or GPB distributions between them were statistically significant $(p<0.001)$. The prevalence of MDR/ XDR strains in GNB infections was much higher in the SBP group than in the bacterascites group ( $p=0.002)$. Interestingly, in patients with bacterascites, the percentage of Enterococcus spp. isolates were higher in nosocomial infections than in healthcare-associated and in community-acquired infections. However, Streptococcus spp. were detected more common in the community-acquired subgroup than in the healthcare-associated and nosocomial subgroups $(p<0.05)$. Other bacteria distributions among the three subgroups were not statistical significance (Supplementary Table 2, only online).

The majority of GNB infections were caused by E. coli (26.2\%) and by K. pneumoniae (7.5\%). GPB included Staphylococcus spp. (17.1\%), Enterococcus spp. (12.7\%), Streptococcus spp. (10.1\%), and others. Candida species accounted for $2.0 \%$ (9/455) of all strains (Supplementary Table 3, only online). ESBL-producing E. coli and K. pneumoniae were isolated in 72 (15.8\%) out of 455 strains and presented less frequently in patients with community-acquired infections (12.1\%) than in those with healthcare-

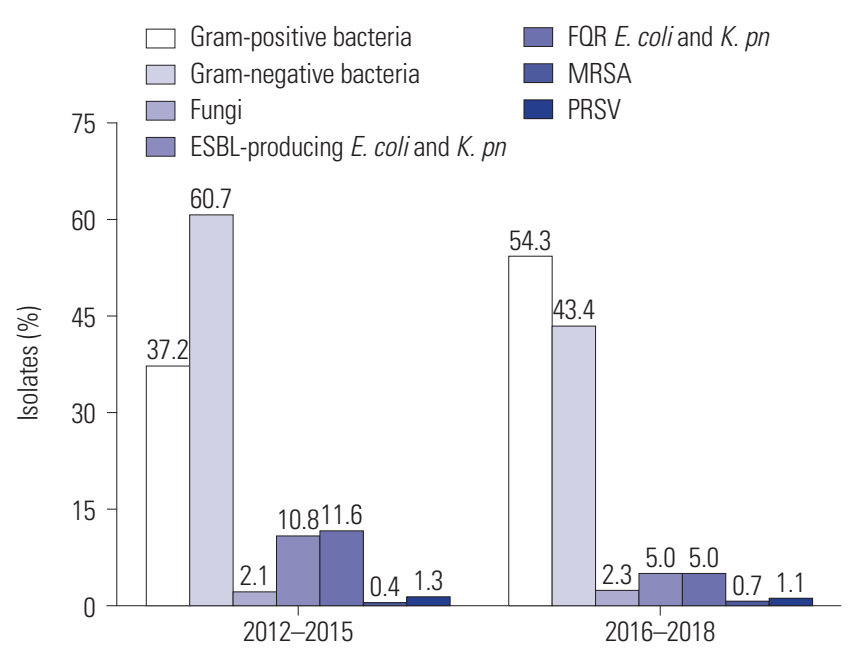

Fig. 2. Trends in microorganism and multidrug-resistant isolates distributions between 2012-2015 and 2016-2018. ESBL, extended-spectrum $\beta$-lactamase; FOR, fluoroquinolone-resistant; MRSA, methicillin-resistant Staphylococcus aureus, PRSV, penicillin-resistant Streptococcus viridans.

associated (17.2\%) and nosocomial infections (16.8\%). Carbapenem-resistant GNB infections were more frequent in the healthcare-associated and nosocomial groups than in the community-acquired group ( $p=0.005$ ) (Supplementary Table 4, only online).

Antibiotic susceptibility analysis

Antibiotic susceptibility of GNB, including E. coli and K. pneu- 
Jing Liu, et al.

Table 2. Distributions of Resistance Microorganisms between Survivors and Non-Survivors

\begin{tabular}{|c|c|c|c|c|}
\hline Microorganism distribution & All $(n=455)$ & Survivors ( $\mathrm{n}=329$ ) & Non-survivors ( $n=126$ ) & $p$ value \\
\hline GNB & $238(52.3)$ & $168(51.1)$ & $70(55.6)$ & 0.391 \\
\hline Escherichia coli (FQR) & $68(14.9)$ & $43(13.1)$ & $25(19.8)$ & 0.070 \\
\hline Escherichia coli (ESBL) & $61(13.4)$ & $39(11.9)$ & $22(17.5)$ & 0.116 \\
\hline Klebsiella pneumoniae (FOR) & $8(1.8)$ & $5(1.5)$ & $3(2.4)$ & 0.691 \\
\hline Klebsiella pneumoniae (ESBL) & $11(2.4)$ & $7(2.1)$ & $4(3.2)$ & 0.506 \\
\hline Acinetobacter baumannii (XDR) & $3(0.7)$ & 0 & $3(2.4)$ & 0.021 \\
\hline Enterobacteriaceae (CRE) & $2(0.4)$ & 0 & $2(1.6)$ & 0.076 \\
\hline MDR GNB* & $76(16.7)$ & $51(15.5)$ & 25 (19.8) & 0.267 \\
\hline XDR GNB* & $6(1.3)$ & $1(0.3)$ & $5(4.0)$ & 0.007 \\
\hline GPB & $207(45.5)$ & $156(47.4)$ & $51(40.5)$ & 0.183 \\
\hline Staphylococci (MRSCoN) & $37(8.1)$ & $30(9.1)$ & $7(5.6)$ & 0.213 \\
\hline Staphylococcus aureus (MRSA) & $5(1.1)$ & $3(0.9)$ & $2(1.6)$ & 0.620 \\
\hline Streptococcus viridans (PRSV) & $11(2.4)$ & $8(2.4)$ & $3(2.4)$ & $>0.999$ \\
\hline MDR GPB ${ }^{\dagger}$ & $20(4.4)$ & $13(4.0)$ & $7(5.6)$ & 0.455 \\
\hline Fungi & $10(2.2)$ & $5(1.5)$ & $5(4.0)$ & 0.149 \\
\hline
\end{tabular}

FQR, fluoroquinolone-resistant; ESBL, extended-spectrum $\beta$-lactamase; XDR, extensively drug resistant; CRE, carbapenemase resistant Enterobacteriaceae; MRSCoN, methicillin resistant coagulase-negative Staphylococcus; MRSA, methicillin-resistant Staphylococcus aureus; PRSV, penicillin-resistant Streptococcus viridans; GNB, gram-negative bacteria; GPB, gram-positive bacteria; MDR GNB, multidrug resistant GNB; XDR GNB, extensively drug resistant GNB. Categorical variables: number (percentage).

${ }^{*} \mathrm{MDR}$ and XDR GNB including Enterobacteriaceae, Pseudomonas aeruginosa; Acinetobacter spp. ${ }^{\dagger} \mathrm{MDR}$ GPB including Enterococcus spp. and Staphylococcus aureus.

moniae, was investigated with respect to susceptibility to 18 different antibiotics covering seven main groups. The E. coli and K. pneumoniae isolates were highly sensitive to meropenem, imipenem, piperacillin/tazobactam, and amikacin, regardless of their ESBL status. Similar results were observed among community-acquired, healthcare-associated, and nosocomial infections; however, non-ESBL-producing agents also showed high sensitivity to third-generation cephalosporins and aztreonam (Table 3).

Antibiotic susceptibility of GPB, including Staphylococcus, Streptococcus viridans, and Enterococcus, was determined. The susceptibility of MRSA to fluoroquinolone antibiotics, erythromycin, clindamycin, sulfamethoxazole, and trimethoprim was weaker than that in methicillin-sensitive isolates. However, methicillin-resistant isolates showed good sensitivity to vancomycin and linezolid among community-acquired, healthcare-associated, and nosocomial infections. PRSV were highly susceptible to other antimicrobials, except for erythromycin, clindamycin, tetracycline, and levofloxacin. In addition, similar to other GPB, both Enterococcus faecium and Enterococcus faecalis were also sensitive to vancomycin and linezolid (Table 4).

\section{Predictors of 28-day mortality in patients with acute decompensated cirrhosis with culture-positive SAI} In univariate analysis, days of hospital stay before culture-positive SAI, prevalence of XDR isolates, severe hepatic encephalopathy (HE) (grade 2-4), upper gastrointestinal bleeding (UGB), WBC count, neutrophil count, aspartate transaminase (AST), alanine aminotransferase (ALT), serum creatinine, total bilirubin, INR, and Model for End-Stage Liver Disease score (MELD) and MELD-Na scores were significantly correlated with outcomes of patients with acute decompensated cirrhosis who developed culture-positive SAI. Multivariate analysis demonstrated that mortality variables were independently associated with days of hospital stay before culture-positive SAI, UGB, WBC count, INR, ALT, serum creatinine, and total bilirubin (Table 5).

\section{DISCUSSION}

The prevalence of MDR pathogens and antibiotic strategies were identified in patients with acute decompensated cirrhosis with culture-positive SAI in our multicenter retrospective study. To the best of our knowledge, this is the most comprehensive and largest contemporary epidemiological survey for this group of patients in China.

Our study indicated that GNB remain the primary pathogens; meanwhile, an increasing prevalence of GPB in SAI was observed. E. coli and K. pneumoniae represented the highest GNB infections, and Staphylococcus was the dominant GPB. These results were different from several single-center studies, which demonstrated Streptococcus spp. and Enterococcus spp. as the most frequently isolated GPB. ${ }^{22,23}$ Historically, GNB have been isolated in the majority of patients with HBV-related acute decompensated cirrhosis complicated with SAI. ${ }^{22,24}$ However, in this study, GPB were found to be the predominant pathogens. Particularly, an increasing rate of infections caused by GPB and resistant microorganisms had been demonstrated in culturepositive cases of SAI. ${ }^{8,12}$ The prevalence of GPB in our study was lower than that reported (55\%) in a recent study; ${ }^{12}$ however, the 
Table 3. In Vitro Susceptibility Rates (\%) of Gram-Negative Isolates

\begin{tabular}{|c|c|c|c|c|c|c|}
\hline \multirow{3}{*}{ Antibiotics } & \multicolumn{6}{|c|}{ Escherichia coli and Klebsiella pneumoniae } \\
\hline & \multicolumn{2}{|c|}{ Community-acquired } & \multicolumn{2}{|c|}{ Healthcare-associated } & \multicolumn{2}{|c|}{ Nosocomial } \\
\hline & ESBL+ & ESBL- & ESBL+ & ESBL- & ESBL+ & ESBL- \\
\hline AMP & 0.0 & 35.0 & 0.0 & 15.2 & 0.0 & 37.0 \\
\hline PIP & 0.0 & 62.5 & 0.0 & 60.0 & 0.0 & 46.2 \\
\hline SAM & 27.3 & 66.7 & 12.5 & 63.3 & 8.3 & 65.4 \\
\hline TZP & 92.3 & 95.0 & 84.0 & 97.0 & 83.3 & 92.3 \\
\hline CZO & 0.0 & 50.0 & 0.0 & 69.2 & 0.0 & 66.7 \\
\hline CAZ & 46.2 & 100 & 29.6 & 100 & 41.9 & 100 \\
\hline FEP & 38.5 & 100 & 36.0 & 100 & 29.0 & 100 \\
\hline ATM & 18.2 & 95.0 & 20.0 & 100 & 33.3 & 96.2 \\
\hline AMK & 81.8 & 100 & 84.0 & 94.1 & 87.1 & 100 \\
\hline GEN & 61.5 & 80.0 & 36.0 & 74.2 & 43.3 & 65.4 \\
\hline IPM & 100 & 100 & 96.3 & 100 & 93.5 & 100 \\
\hline MEM & 100 & 100 & 100 & 100 & 92.9 & 100 \\
\hline CIP & 23.1 & 75.0 & 29.6 & 70.6 & 31.3 & 55.6 \\
\hline LVX & 23.1 & 75.0 & 29.6 & 75.8 & 31.3 & 55.6 \\
\hline SXT & 23.1 & 57.9 & 24.0 & 51.6 & 25.0 & 50.0 \\
\hline
\end{tabular}

AMP, ampicillin; PIP, piperacillin; SAM, ampicillin/sulbactam; TZP, piperacillin/tazobactam; CZO, cefazolin; CAZ, ceftazidime; FEP, cefepime; ATM, aztreonam; AMK, amikacin; GEN, gentamicin; IPM, imipenem; MEM, meropenem; CIP, ciprofloxacin; LVX, levofloxacin; SXT, sulfamethoxazole and trimethoprim; ESBL, extended-spectrum $\beta$-lactamase.

Table 4. In Vitro Susceptibility Rates (\%) of Gram-Positive Isolates

\begin{tabular}{lccccccccccccc}
\hline Gram-positive isolates & Type & PEN & CIP & LVX & MFX & TET & ERY & CLI & LNZ & VAN & GEN & RIF & SXT \\
\hline Staphylococcus & & & & & & & & & & & & \\
Community-acquired & MRS & 0.0 & 70.0 & 60.0 & 60.0 & 76.9 & 23.1 & 38.5 & 100 & 100 & 84.6 & 92.3 & 45.5 \\
& MSS & 33.3 & 88.9 & 87.5 & 100 & 66.7 & 45.5 & 70.0 & 100 & 100 & 100 & 100 & 81.8 \\
\multirow{2}{*}{ Healthcare-associated } & MRS & 0.0 & 62.5 & 62.5 & 57.1 & 77.8 & 10.0 & 40.0 & 100 & 100 & 70.0 & 77.8 & 70.0 \\
& MSS & 20.0 & 92.9 & 100 & 100 & 83.3 & 21.4 & 80.0 & 100 & 100 & 100 & 100 & 92.3 \\
\multirow{2}{*}{ Nosocomial } & MRS & 10.5 & 41.2 & 44.4 & 47.1 & 88.2 & 10.5 & 47.4 & 100 & 94.4 & 77.8 & 100 & 70.6 \\
& MSS & 0.0 & 66.7 & 71.4 & 83.3 & 87.5 & 25.0 & 62.5 & 100 & 100 & 71.4 & 87.5 & 62.5 \\
\hline \multirow{2}{*}{ Enterococcus faecium } & Type & PEN & CIP & LVX & MFX & TET & ERY & CLI & LNZ & VAN & GEN & RIF & SXT \\
Enterococcus faecalis & - & 5.3 & 7.4 & 0.0 & - & 50.0 & 3.6 & - & 93.3 & 100 & 40.7 & - & - \\
\hline \multirow{2}{*}{ Streptococcus viridans } & - & 100 & 66.7 & 87.5 & - & 25.0 & 20.0 & - & 100 & 100 & 42.9 & - & - \\
\hline
\end{tabular}

PEN, penicillin; CIP, ciprofloxacin; LVX, levofloxacin; MFX, moxifloxacin; TET, tetracycline; ERY, erythromycin; CLI, clindamycin; LNZ, linezolid; VAN, vancomycin; GEN, gentamicin; RIF, rifampin; SXT, sulfamethoxazole and trimethoprim; CAZ, ceftazidime; CRO, ceftriaxone; FEP, cefepime; CHL, chloramphenicol; MRS, methicillin-resistant Staphylococcus; MSS, methicillin-sensitive Staphylococcus; PRSV, penicillin-resistant Streptococcus viridans; PSSV, penicillin-sensitive Streptococcus viridans.

increasing trend of GPB infections in culture-positive SAI patients with acute decompensated cirrhosis remains obvious and may be attributed to an exposure to healthcare services and previous antibiotics uses before admission, especially a widespread use of prophylactic antibiotics against GNB in patients with culture-positive SAI. Thus, antibiotics used to manage GPB infections should also be considered in future empirical therapies.

An increase in MDR and XDR bacteria in patients with SBP has been reported. ${ }^{25}$ As shown in our study, the prevalence of MDR and/or XDR pathogens in patients with culture-positive SBP or bacterascites were $29.6 \%$ and $18.2 \%$, respectively. These percentages were higher than those reported among patients with $\mathrm{SBP}{ }^{19,26,27}$ but lower than that reported (25\%) among patients with bacterascites by Oey, et al. ${ }^{8}$ These results emphasized an urgent need to control the increase of drug resistant bacteria in patients with SAI. Additionally, our results indicated that MDR organisms are more prevalent in nosocomial and healthcare-associated patients with SAI than in communityacquired patients. The prevalence of ESBL-producing organisms in community-acquired infections was much higher than that reported, ${ }^{28}$ which may be attributed to the extensive use of thirdgeneration cephalosporins in China. Furthermore, patients with 
Jing Liu, et al.

Table 5. Predictors of 28-Day Mortality in Univariate and Multivariate Analysis of Cirrhotic Patients with Culture-Positive SAI

\begin{tabular}{|c|c|c|c|c|}
\hline \multirow{2}{*}{ Predictors } & \multicolumn{2}{|c|}{ Univariate analysis } & \multicolumn{2}{|c|}{ Multivariate analysis } \\
\hline & HR (Cl 95\%) & $p$ value & HR (CI 95\%) & $p$ value \\
\hline Age & $0.998(0.981-1.015)$ & 0.792 & & \\
\hline Sex & $0.975(0.620-1.535)$ & 0.914 & & \\
\hline Exposure to health care services & $1.294(0.851-1.966)$ & 0.228 & & \\
\hline Previous antibiotics use & $1.248(0.704-2.212)$ & 0.447 & & \\
\hline Days of hospital stay before SAI & $0.956(0.916-0.998)^{*}$ & $0.039 *$ & $0.925(0.881-0.972)^{*}$ & $0.002^{*}$ \\
\hline Prevalence of XDR isolates & $3.441(1.082-10.946)^{*}$ & $0.036^{*}$ & $2.410(0.702-8.273)$ & 0.162 \\
\hline Prevalence of MDR isolates & 1.369 (0.857-2.187) & 0.189 & & \\
\hline BSIs & $0.722(0.292-1.782)$ & 0.479 & & \\
\hline Pneumonia & 1.295 (0.809-2.073) & 0.282 & & \\
\hline UTIS & 1.583 (0.499-5.030) & 0.436 & & \\
\hline HE (Grade 2-4) & $2.372(1.359-4.139)^{*}$ & $0.002^{*}$ & 1.568 (0.839-2.930) & 0.158 \\
\hline UGB & $2.072(1.125-3.819)^{*}$ & $0.019^{*}$ & $2.676(1.382-5.180)^{*}$ & $0.003^{*}$ \\
\hline Serum WBC & $1.065(1.036-1.094)^{*}$ & $<0.001^{*}$ & $1.053(1.020-1.086)^{*}$ & $0.001^{*}$ \\
\hline Neutrophil & $1.046(1.031-1.062)^{*}$ & $<0.001^{*}$ & & \\
\hline PLT & 0.999 (0.996-1.002) & 0.412 & & \\
\hline AST & $1.001(1.001-1.002)^{*}$ & $<0.001^{*}$ & $0.999(0.997-1.000)$ & 0.081 \\
\hline ALT & $1.002(1.001-1.003)^{*}$ & $<0.001^{*}$ & $1.002(1.000-1.003)^{*}$ & $0.025^{*}$ \\
\hline Albumin & 0.965 (0.930-1.001) & 0.059 & $0.965(0.927-1.006)$ & 0.091 \\
\hline Serum creatinine & $1.680(1.457-1.938)^{*}$ & $<0.001^{*}$ & $1.508(1.292-1.761)^{*}$ & $<0.001^{*}$ \\
\hline Total bilirubin & $1.073(1.055-1.091)^{*}$ & $<0.001^{*}$ & $1.059(1.039-1.079)^{*}$ & $<0.001^{*}$ \\
\hline INR & $1.284(1.202-1.372)^{*}$ & $<0.001^{*}$ & $1.295(1.141-1.470)^{*}$ & $<0.001^{*}$ \\
\hline MELD score & $1.124(1.101-1.147)^{*}$ & $<0.001^{*}$ & & \\
\hline MELD-Na score & $1.170(1.136-1.206)^{*}$ & $<0.001^{*}$ & & \\
\hline
\end{tabular}

SAI, spontaneous ascitic infection; BSIs, blood stream infections; UTIs, urinary tract infections; HE (Grade 2-4), hepatic encephalopathy (Grade 2-4); UGB, upper gastrointestinal bleeding; $\mathrm{HR}$, hazard ratio; $\mathrm{Cl}$, confidence interval.

MELD scores, MELD-Na scores and neutrophil were not included in the multivariate model to avoid potential collinearities.

${ }^{*} p$-values $<0.05$ and corresponding HR are indicated.

healthcare-associated infections had a higher 28-day mortality than patients with community-acquired SAI. Thus, attention should be paid to patients with healthcare-associated and nosocomial SAI.

Third-generation cephalosporins, piperacillin-tazobactam, and carbapenems are recommended in the recent EASL guidelines for the treatment of patients with decompensated cirrhosis with SBP. ${ }^{13}$ However, the increase in XDR and MDR strains challenge the recommended antibiotics from the guidelines. Our findings showed that ESBL-producing E. coli and K. pneumoniae accounted for $15.8 \%$. A significant number of ESBLproducing strains exhibited low sensitivity to third-generation cephalosporins and/or quinolones, especially pathogens in community-acquired infections. In the present study, ceftazidime, cefepime, aztreonam, and amikacin were recommended for first-line antibiotic treatment for non-MDR GNB infections. Considering the high mortality, piperacillin/tazobactam and carbapenems should be considered for MDR GNB infections in community-acquired SAI and healthcare-associated or nosocomial SAI, respectively, which is in accordance with EASL guidelines. However, unlike the ESAL guidelines that recommend carbapenems combined with glycopeptides or daptom- ycin or linezolid, vancomycin or linezolid should be considered for GPB infections, regardless of drug-resistance status and type of infections.

Previous studies have reported on independent risk factors in patients with SBP; $;^{1,29}$ however, no study has evaluated the specific risk factors for culture-positive SAI in patients with acute decompensated cirrhosis. In our series, severe HE, UGB, WBC count, serum creatinine, and INR were independently associated with 28-day all-cause mortality, and these results emphasized the importance of the timely treatment in complications. More importantly, reducing days of hospital stay before SAI, which meant early diagnosis and treatment, was also closely related to 28-day mortality.

Our study had several limitations. First, our study aimed to analyze the microbiological characteristics of patients with culture-positive SAI, thus culture-negative patients with SAI were excluded from our study. Second, we included Staphylococcus because this strain could be isolated from both the blood and ascitic fluid samples obtained from one patient. Furthermore, exogenous contamination related to invasive procedures, such as traumatic paracentesis, was unlikely to account for any of the culture-positive SAI cases, and no effective methods to distin- 
guish contaminants from pathogens because SAI may be caused by Staphylococcus with low virulence in susceptible patients. Finally, a few clinical results, such as AST and ALT, were missing in patient records, but did not significantly impact the results.

In conclusion, GNB were the most common pathogens, with a growing proportion of SAIs caused by GPB and MDR/XDR GNB. Healthcare-associated and nosocomial infections showed a high prevalence of MDR organisms and exhibited differences in 28-day mortality, compared to community-acquired infections. Carbapenems, piperacillin/tazobactam, and amikacin were more suitable for the treatment of culture-positive SAI with GNB infections, whereas vancomycin and linezolid were the best choices for GPB infections. All identified independent risk factors could be utilized to develop a multiple clinical risk evaluation system to help clinicians identify the highest-risk subsets of patients.

\section{ACKNOWLEDGEMENTS}

We thank the Chinese Acute-on-Chronic Liver Failure Consortium members and participants for their contributions to this study: Zhang Yanan, Department of Hepatology, The First Hospital of Jilin University; Huang Yunyi, Center of Integrative Medicine, Beijing Ditan Hospital, Capital Medical University; Zou Ying, Department of Liver Intensive Care Unit, Shanghai Public Health Clinical Centre, Fudan University; Li Beiling, Hepatology Unit, Department of Infectious Diseases, Nanfang Hospital, Southern Medical University; Zheng Yixiang, Department of Infectious Disease, Hunan Key Laboratory of Viral Hepatitis, Xiangya Hospital, Central South University; Wang Chen, Department of Infectious Disease, Taihe Hospital, Hubei University of Medicine; Zhu Yanfang, Liver Disease Center, First Affiliated Hospital of Xinjiang Medical University; Xiang Xiaomei, Department of Infectious Diseases, Southwest Hospital, Third Military Medical University (Army Medical University); Li Jing, and Zhang Yan, Department of Infectious Diseases and Hepatology, The Second Hospital of Shandong University; Department of Gastroenterology, Ren Ji Hospital, School of Medicine, Shanghai Jiao Tong University.

This work was supported by the National Natural Science Foundation of China (grant numbers 81271884 and 81461130019), the National Key Research and Development Program of China (grant numbers 2017YFC0908100 and 2017YFC0908104), and the National Science and Technology Major Project (grant numbers 2018ZX10723203 and 2018ZX10302206).

\section{AUTHOR CONTRIBUTIONS}

Conceptualization: Jing Liu, Yanhang Gao, Xianbo Wang, and Xin Zheng. Data curation: Jing Liu. Formal analysis: Jing Liu. Funding acquisition: Xin Zheng, Hai Li, and Jinjun Chen. Investigation: Zhiping Qian, Jinjun Chen, Yan Huang, Zhongji Meng, Xiaobo Lu, Guohong Deng, Feng Liu, and Hai Li. Methodology: Zhiguo Zhang. Project administration: Jing Liu. Resources: Zhiping Qian, Jinjun Chen, Yan
Huang, Zhongii Meng, Xiaobo Lu, Guohong Deng, Feng Liu, and Hai Li. Software: Zhiguo Zhang. Supervision: Jing Liu and Xin Zheng. Validation: Jing Liu and Xin Zheng. Visualization: Jing Liu. Writingoriginal draft: Jing Liu and Xin Zheng. Writing—review \& editing: Jing Liu and Xin Zheng. Approval of final manuscript: all authors.

\section{ORCID iDs}

Jing Liu https://orcid.org/0000-0002-4283-4375 Yanhang Gao https://orcid.org/0000-0002-7248-8290 Xianbo Wang https://orcid.org/0000-0002-3593-5741 Zhiping Qian https://orcid.org/0000-0003-4641-3348 Jinjun Chen https://orcid.org/0000-0003-4275-9149 Yan Huang https://orcid.org/0000-0002-4747-3740 Zhongji Meng https://orcid.org/0000-0003-0401-535X Xiaobo Lu https://orcid.org/0000-0001-5532-7551 Guohong Deng https://orcid.org/0000-0003-1263-7220 Feng Liu https://orcid.org/0000-0001-7060-3710 Zhiguo Zhang https://orcid.org/0000-0002-5081-4382 Hai Li https://orcid.org/0000-0003-4174-9758 Xin Zheng https://orcid.org/0000-0001-6564-7807

\section{REFERENCES}

1. Schwabl P, Bucsics T, Soucek K, Mandorfer M, Bota S, Blacky A, et al. Risk factors for development of spontaneous bacterial peritonitis and subsequent mortality in cirrhotic patients with ascites. Liver Int 2015;35:2121-8.

2. Garcia-Tsao G. Bacterial infections in cirrhosis. Can J Gastroenterol 2004;18:405-6.

3. Leber B, Spindelboeck W, Stadlbauer V. Infectious complications of acute and chronic liver disease. Semin Respir Crit Care Med 2012; 33:80-95.

4. Moreau R, Jalan R, Gines P, Pavesi M, Angeli P, Cordoba J, et al. Acute-on-chronic liver failure is a distinct syndrome that develops in patients with acute decompensation of cirrhosis. Gastroenterology 2013;144:1426-37.

5. Kaymakoglu S, Eraksoy H, Okten A, Demir K, Calangu S, Cakaloglu $\mathrm{Y}$, et al. Spontaneous ascitic infection in different cirrhotic groups: prevalence, risk factors and the efficacy of cefotaxime therapy. Eur J Gastroenterol Hepatol 1997;9:71-6.

6. Rimola A, García-Tsao G, Navasa M, Piddock LJ, Planas R, Bernard $\mathrm{B}$, et al. Diagnosis, treatment and prophylaxis of spontaneous bacterial peritonitis: a consensus document. International Ascites Club. J Hepatol 2000;32:142-53.

7. Arvaniti V, D'Amico G, Fede G, Manousou P, Tsochatzis E, Pleguezuelo M, et al. Infections in patients with cirrhosis increase mortality four-fold and should be used in determining prognosis. Gastroenterology 2010;139:1246-56.

8. Oey RC, van Buuren HR, de Jong DM, Erler NS, de Man RA. Bacterascites: a study of clinical features, microbiological findings, and clinical significance. Liver Int 2018;38:2199-209.

9. Dellinger RP, Levy MM, Carlet JM, Bion J, Parker MM, Jaeschke R, et al. Surviving Sepsis Campaign: international guidelines for management of severe sepsis and septic shock: 2008. Crit Care Med 2008;36:296-327.

10. Ricart E, Soriano G, Novella MT, Ortiz J, Sàbat M, Kolle L, et al. Amoxicillin-clavulanic acid versus cefotaxime in the therapy of bacterial infections in cirrhotic patients. J Hepatol 2000;32:596-602.

11. European Association for the Study of the Liver. EASL clinical practice guidelines on the management of ascites, spontaneous bacterial peritonitis, and hepatorenal syndrome in cirrhosis. J Hepatol 
2010;53:397-417.

12. Alexopoulou A, Papadopoulos N, Eliopoulos DG, Alexaki A, Tsiriga A, Toutouza $M$, et al. Increasing frequency of gram-positive cocci and gram-negative multidrug-resistant bacteria in spontaneous bacterial peritonitis. Liver Int 2013;33:975-81.

13. European Association for the Study of the Liver. EASL Clinical Practice Guidelines for the management of patients with decompensated cirrhosis. J Hepatol 2018;69:406-60.

14. Goossens H, Ferech M, Vander Stichele R, Elseviers M; ESAC Project Group. Outpatient antibiotic use in Europe and association with resistance: a cross-national database study. Lancet 2005;365:579-87.

15. Hung TH, Tsai CC, Hsieh YH, Tsai CC, Tseng CW, Tseng KC. The effect of the first spontaneous bacterial peritonitis event on the mortality of cirrhotic patients with ascites: a nationwide populationbased study in Taiwan. Gut Liver 2016;10:803-7.

16. Karvellas CJ, Abraldes JG, Arabi YM, Kumar A; Cooperative Antimicrobial Therapy of Septic Shock (CATSS) Database Research Group. Appropriate and timely antimicrobial therapy in cirrhotic patients with spontaneous bacterial peritonitis-associated septic shock: a retrospective cohort study. Aliment Pharmacol Ther 2015;41:747-57.

17. Kim JJ, Tsukamoto MM, Mathur AK, Ghomri YM, Hou LA, Sheibani S, et al. Delayed paracentesis is associated with increased inhospital mortality in patients with spontaneous bacterial peritonitis. Am J Gastroenterol 2014;109:1436-42.

18. Lutz P, Nischalke HD, Krämer B, Goeser F, Kaczmarek DJ, Schlabe $\mathrm{S}$, et al. Antibiotic resistance in healthcare-related and nosocomial spontaneous bacterial peritonitis. Eur J Clin Invest 2017;47:44-52.

19. Salerno F, Borzio M, Pedicino C, Simonetti R, Rossini A, Boccia S, et al. The impact of infection by multidrug-resistant agents in patients with cirrhosis. A multicenter prospective study. Liver Int 2017;37: 71-9.

20. Magiorakos AP, Srinivasan A, Carey RB, Carmeli Y, Falagas ME, Giske CG, et al. Multidrug-resistant, extensively drug-resistant and pandrug-resistant bacteria: an international expert proposal for interim standard definitions for acquired resistance. Clin Microbiol Infect 2012;18:268-81.

21. CLSI. Performance standards for antimicrobial susceptibility testing. 28th ed. CLSI supplement M100. Wayne (PA): Clinical and Laboratory Standards Institute; 2018.

22. Ning NZ, Li T, Zhang JL, Qu F, Huang J, Liu X, et al. Clinical and bacteriological features and prognosis of ascitic fluid infection in Chinese patients with cirrhosis. BMC Infect Dis 2018;18:253.

23. Friedrich K, Nüssle S, Rehlen T, Stremmel W, Mischnik A, Eisenbach C. Microbiology and resistance in first episodes of spontaneous bacterial peritonitis: implications for management and prognosis. J Gastroenterol Hepatol 2016;31:1191-5.

24. Cheong HS, Kang CI, Lee JA, Moon SY, Joung MK, Chung DR, et al. Clinical significance and outcome of nosocomial acquisition of spontaneous bacterial peritonitis in patients with liver cirrhosis. Clin Infect Dis 2009;48:1230-6.

25. Salerno F, Cazzaniga M. Therapeutic strategies and emergence of multiresistant bacterial strains. Intern Emerg Med 2010;5 Suppl 1: S45-51.

26. Fernández J, Prado V, Trebicka J, Amoros A, Gustot T, Wiest R, et al. Multidrug-resistant bacterial infections in patients with decompensated cirrhosis and with acute-on-chronic liver failure in Europe. J Hepatol 2019;70:398-411.

27. Piano S, Singh V, Caraceni P, Maiwall R, Alessandria C, Fernandez J, et al. Epidemiology and effects of bacterial infections in patients with cirrhosis worldwide. Gastroenterology 2019;156:1368-80.

28. Tandon P, Delisle A, Topal JE, Garcia-Tsao G. High prevalence of antibiotic-resistant bacterial infections among patients with cirrhosis at a US liver center. Clin Gastroenterol Hepatol 2012;10:1291-8.

29. Ariza X, Castellote J, Lora-Tamayo J, Girbau A, Salord S, Rota R, et al. Risk factors for resistance to ceftriaxone and its impact on mortality in community, healthcare and nosocomial spontaneous bacterial peritonitis. J Hepatol 2012;56:825-32. 